\title{
Uso de imagens de radar para o cálculo da produção primária de plantas aquáticas nas várzeas da Amazônia.
}

\author{
Maycira COSTA ${ }^{1}$
}

\begin{abstract}
RESUMO
A área da planície de inundação da Amazônia é estimada em $300000 \mathrm{~km}^{2}$ e sua produtividade primária em 1,17 x $10^{14} \mathrm{~g} \mathrm{C} \mathrm{yr}^{-1}$. Deste total de área e produtividade, estimativas sugerem que $43 \%$ e $62 \%$, respectivamente, são atribuídos às plantas aquáticas. Estas estimativas variam de acordo com o pulso de inundação. Por exemplo, durante o período de seca as plantas terrestres (herbáceas) geralmente ocupam áreas que apresentam plantas aquáticas na cheia. Aárea e a produtividade destes ecossistemas são informações essenciais para a compreensão da dinâmica biogeoquímica da Amazônia. Imagens de satélites (radar) combinadas com amostragem de campo foram utilizadas para estimar a biomassa e mapear a área de cobertura de plantas aquáticas emergentes para calcular a produção primária de plantas aquáticas na várzea do baixo Amazonas. A combinação de bandas $\mathrm{Ce}$ $\mathrm{L}$ forneceu a melhor correlação $(\mathrm{r}=0,82)$ e um ponto de saturação de biomassa intermediário $\left(620 \mathrm{gm}^{-2}\right)$ para estimar biomassa aérea. O método de segmentação e classificação por região foi utilizado para classificar combinações de bandas C e L para cada período de nível de água, e forneceu uma precisão de mapeamento maior que 95\% para determinação espacial de áreas cobertas por plantas aquáticas. Combinando a distribuição espacial de plantas aquáticas, o modelo para estimativa de biomassa aérea e a porcentagem de biomassa submersa, estimou-se espacialmente uma produção primária líquida anual de $1.9 \times 10^{12} \mathrm{~g}$ C $\mathrm{yr}^{-1}( \pm 28 \%)$ para as plantas aquáticas em uma área de $394 \mathrm{~km}^{2}$.
\end{abstract}

\section{PALAVRA CHAVES}

Imagens de satélite radar, produção primária, plantas aquáticas, várzea.

\section{Use of radar imagery for estimating net primary productivity of aquatic vegetation in the Amazon floodplain.}

\begin{abstract}
Estimates suggest that $43 \%$ and $63 \%$ of the $300,000 \mathrm{~km}^{2}$ of Amazon floodplain area and of the $1.17 \times 10^{14} \mathrm{~g}$ C $y r^{1}$ of primary productivity, respectively, are attributed to the aquatic vegetation. These numbers change according to the flood pulse. For instance, in the dry season terrestrial herbaceous plants generally occupy areas that have lost their aquatic vegetation. The areal extent and productivity of these ecosystems are essential to construe even an initial understanding of the biogeochemistry of the Amazon. Field measurements were combined with synthetic aperture radar images to evaluate the use of RADARSAT and JERS1 for estimating biomass changes and mapping of aquatic vegetation, and subsequently estimating the net primary productivity of aquatic vegetation in the lower Amazon. The combination of $C$ and $L$ bands provides the best correlation $(r=0.82)$ and an intermediate saturation point $\left(620 \mathrm{gm}^{2}\right)$ for estimating above water biomass of aquatic vegetation. A combination of RADARSAT and JERS-1 images from each water period was classified using a region growing algorithm, and yielded accuracy bigher than 95\% for the seasonal vegetated areas of the floodplain. The combination of the seasonal mapped area of aquatic vegetation with the statistical SAR-algorithm for estimating above water biomass and the percentage of below water biomass yielded a total annual NPP of $1.9 \times 10^{12} \mathrm{gCyr}^{-1}( \pm 28 \%)$ for aquatic vegetation.
\end{abstract}

\section{KEYWORDS}

Radar satellite imagery, primary production, aquatic vegetation, várzea.

\footnotetext{
${ }^{1}$ University of Victoria, Canada. Department of Geography. P.O. Box 3050, Victoria, BC, Canada. V8W 3P5. email:maycira@office.geog.uvic.ca Instituto Nacional de Pesquisas Espaciais - INPE - Divisão de Sensoriamento Remoto
} 


\section{ACTA \\ AMAZONICA}

USO DE IMAGENS DE RADAR PARA O CÁLCULO DA PRODUCÃO PRIMÁRIA DE PLANTAS AQUÁTICAS NAS VÁRZEAS DA AMAZÔNIA.

\section{INTRODUÇÃO}

A planície de inundação do Rio Amazonas faz parte da vasta área de regiões alagadas tropicais e subtropicais, as quais compõem aproximadamente 56\% das áreas alagadas do globo (Mitsch \& Gosselink, 2000). Aárea da planície de inundação da Amazônia é estimada em 300 000km² (Junk, 1993) e sua produtividade primária em $1,17 \times 10^{14} \mathrm{~g} \mathrm{C} \mathrm{yr}^{-1}$ (Melack \& Forsberg, 2000). Deste total de área e produtividade, estimativas sugerem que $43 \%$ e $62 \%$, respectivamente, são atribuídos as plantas aquáticas(Melack \& Forsberg, 2000), as quais se proliferam predominantemente na regiao de várzea (Piedade et al. 1991). Esta alta produtividade é decorrente da alta eficiência destas plantas em converterem a energia solar interceptada em biomassa, característica essencial para a sua sobrevivência em um ambiente de contínua variação de nível da água devido ao pulso de inundação (Piedade et al. 1991).

Devido à heterogeneidade espacial da planície de inundação, estimativas mais refinadas de área e produtividade só são possíveis com uma intensa amostragem de campo ou com o uso de imagens de satélite associado à amostragem de campo. A primeira opção se torna inviável devido às dimensões espaciais da Amazônia. A segunda opção é apresentada nesta pesquisa. Outros autores (Potter et al. 1993; Sellers et al. 1995) utilizaram com sucesso dados de satélites ópticos em modelos de estimativa de produção primária em estudos regionais e globais. Contudo, imagens de satélites ópticos sem cobertura de nuvens não estão disponíveis na frequência necessária para a Amazônia. Por exemplo, Novo et al. (1997) utilizaram imagens do satélite Landsat adquiridas durante 10 anos para construir um mosaico sem nuvens da várzea do Amazonas. Para a região geográfica que estamos estudando, em um levantamento de quatro anos de aquisição de imagens Landsat (um total de 38 imagens), somente $3 \%$ das imagens não apresentam nuvens; as outras apresentam aproximadamente $100 \%$ de cobertura de nuvens. Portanto, o uso de imagens de satélite radar é uma das únicas soluções para estudos temporais na Amazônia.

Este trabalho relata um método eficiente que utiliza imagens de satélites radares para mapeamento sazonal e estimativa de produção primária líquida (PPL) de plantas aquáticas na Amazônia.

\section{MÉTODOS}

\section{Modelo para estimativa de PPL usando imagens de radar}

$$
\begin{aligned}
& \text { A PPL foi estimada baseada na seguinte equação: } \\
& \qquad P P=\sum\left\{\left(B_{(t+1)}-B_{t}\right)=\left(L_{(t+1)} \mathbf{x}_{(t+1)}\right]\right.
\end{aligned}
$$

Equação 1

onde $\mathrm{B}_{\mathrm{t}}$ e $\mathrm{B}_{\mathrm{t}+1}$ correspondem à biomassa seca total no começo e final do intervalo temporal de medida e $\mathrm{L}_{\mathrm{t}+1}$ é a porcentagem de biomassa seca perdida durante o mesmo intervalo. Todos os parâmetros são calculados para cada célula de resolução espacial (pixel) da imagem de radar correspondente à área coberta por uma determinada planta aquática. Esta mesma equação foi originalmente utilizada por Junk \& Piedade (1993), porém, os autores basearam-se unicamente em amostragem de campo.

\section{Os parâmetros necessários para o uso da equação 1 são:}

(i)A área espacial ocupada pela plantas aquáticas

(ii)Medidas mensais de biomassa e perda de biomassa

A explicação detalhada da estimativa destes parâmetros utilizando imagens de radar é apresentada nos próximos itens.

\section{Área de estudo}

A área de estudo, Lago Grande de Monte Alegre (LGMA), (Figura 1), está localizada nas coordenadas $2^{\circ} 00^{\prime} \mathrm{S} / 54^{\circ} 00^{\prime} \mathrm{O}$ e $2^{\circ} 30^{\prime} \mathrm{S} / 54^{\circ} 30^{\prime} \mathrm{O}$, próximo à cidade de Santarém. A vegetação predominante na planície de inundação é constituída por grandes bancos de plantas aquáticas herbáceas, arbustos pioneiros, e floresta. Na região de "terra firme" (regiões marginais não alagáveis), a vegetação é composta predominantemente por pequenos campos agrícolas, cerrados e floresta secundária (RADAMBRASIL, 1976). Hymenachne amplexicaulis (rabo de rato), Echinochloa polystachya (canarana) e Paspalum repens (premenbeca) são as espécies dominantes de plantas aquáticas na planície de inundação. Este estudo é focada na espécie $H$. amplexicaulis por ser a mais comum na região (corresponde a $65 \%$ das amostras coletadas). Esta espécie forma grandes bancos mono-específicos na zona litoral do lago, onde a profundidade média é aproximadamente $3,5 \pm 0,5 \mathrm{~m}$ em maio (água alta) e $0 \mathrm{~m}$ em

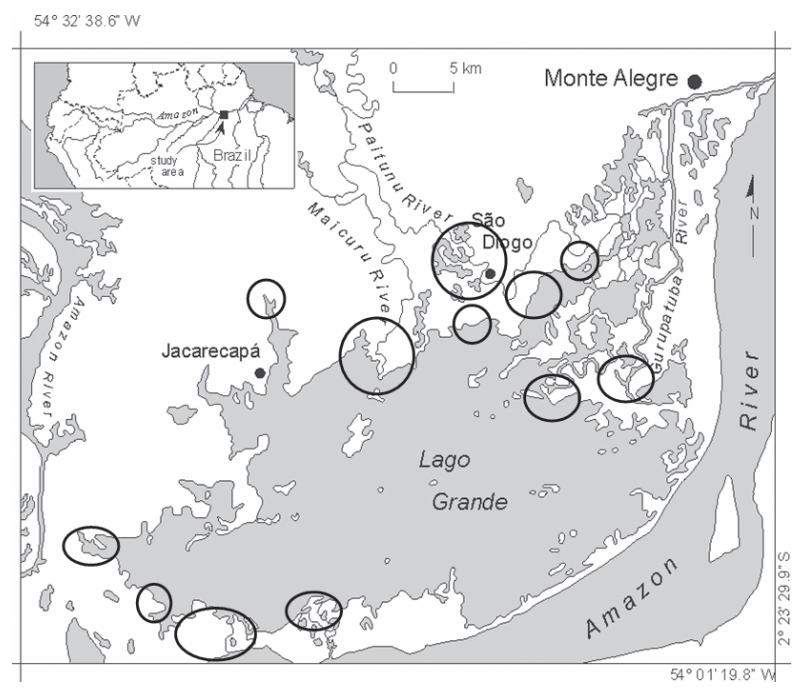

Figura 1 - Área de estudo. Áreas circulares escuras representam as regióes onde o esforço amostral foi concentrado. (Fonte: Costa, 2000). 


\section{ACTA \\ AMAZONICA}

USO DE IMAGENS DE RADAR PARA O CÁLCULO DA PRODUÇÃO PRIMÁRIA DE PLANTAS AQUÁTICAS NAS VÁRZEAS DA AMAZÔNIA. outubro (água baixa). Esta espécie apresenta uma fase terrestre e está distribuída largamente em toda a várzea Amazônica, principalmente nos lagos e áreas susceptíveis à sedimentação (Junk \& Piedade, 1993).

A figura 2 apresenta o ciclo monomodal do Rio Amazonas, na altura de Óbidos, estação mais próxima da área de estudo, onde os níveis mais altos de água ocorrem em maio/junho e os níveis mais baixos em outubro/novembro. As curvas do ciclo hidrológico também mostram variações similares do nível da água para a mesma época do ano para os três anos de aquisição de dados de campo e satélite. Devido a essa variação similar nos níveis de água foi possível assumir que os dados adquiridos (satélite e campo) representam um único ciclo hidrológico contínuo, isto é, estamos assumindo que os dados foram adquiridos durante um ano do ciclo hidrológico. Esta consideração é importante porque a variação temporal da altura da água é de importância fundamental no ciclo de crescimento das plantas aquáticas na Amazônia (Junk \& Piedade, 1997).

\section{Dados de campo}

Um total de cinco campanhas de campo foram realizadas coincidentes com o período de água alta (maio 1996), descida de água (agosto 1996), água baixa (novembro 1996), subida de água (abril 1997), e novamente, água alta (junho 1999). Pontos amostrais foram estabelecidos em regiões de grandes bancos homogêneos de plantas aquáticas utilizando o método de estratificação aleatória (Figura 1 indica as regiões amostrais onde um total de 96 pontos amostrais foram determinados). Nos pontos amostrais foram coletadas amostras para determinação de biomassa aérea e submersa usando um quadrado de $0,25 \mathrm{~m}^{2}$ de área. Outros parâmetros como altura das plantas, espécie dominante, estágio fenológico e profundidade local foram determinados. As amostras de biomassa foram apropriadamente secas e pesadas para determinação de biomassa seca (Dowing \& Anderson, 1985).

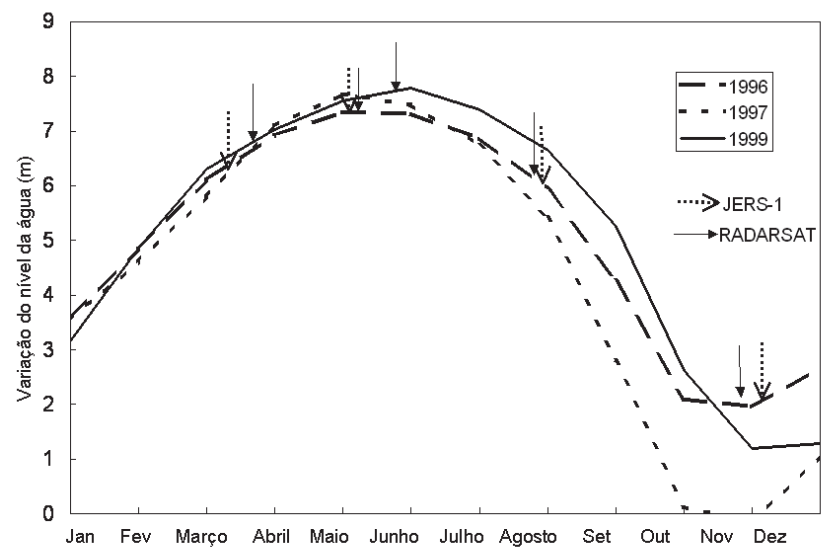

Figure 2 - Variação do nível da água em Óbidos. Setas com traço contínuo e pontilhado representam as data de aquisição de imagens do satélite Radarsat e JERS-1 para a região de estudo. (Fonte: Costa, 2000).
O valor de biomassa seca submersa total foi estimado considerando a biomassa submersa de três plantas individuais, o comprimento de talos submersos individuais, o número de plantas por quadrante e a profundidade local. A correlação entre comprimento das plantas e profundidade local é 0,95 , indicando que a medida de profundidade pode ser utilizada para estimar o comprimento total das hastes submersas (Costa, 2000).

\section{Imagens de satélite}

Imagens dos satélites Radarsat e JERS foram adquiridas coincidentes com as campanhas de campo. As características destas imagens são apresentadas de forma sucinta na tabela 1 . Condições de nenhuma chuva ou pouca chuva foram observadas durante três dias anteriores e durante o período de aquisição das imagens, de forma que as condições meteorológicas não influenciaram a qualidade radiometrica das mesmas.

Dois objetivos determinaram as etapas necessárias para processamento das imagens: (i) estimativa de biomassa a partir de valores de retroespalhamento do radar e (ii) determinação da distribuição espacial de plantas aquáticas a partir das imagens de radar. As etapas de processamento são apresentadas a seguir.

\section{Calibração radiométrica}

O processo de calibração radiométrica implica no uso de constantes determinadas para as imagens do Radarsat e JERS. As equações a seguir foram utilizadas nesta etapa:

$$
\begin{aligned}
& \sigma_{j}^{o}=10 \log _{10}\left[\left(N D_{j}^{2}+A_{o}\right) / A_{g j}\right]+10 \log _{10} \operatorname{sen} \Theta j \text {-Equação } 2 \\
& \mathrm{e} \\
& \sigma_{j}^{\mathrm{o}}=10 \log _{10}\left[N D_{j}^{2}\right]+\mathrm{K} \text { - Equação } 3
\end{aligned}
$$

Onde $\sigma^{\circ}$ é o valor de retroespalhamento calibrado radiometricamente em unidade linear a partir do valor de nível digital (ND), $\mathrm{A}_{\mathrm{o}}$ e $\mathrm{A}_{\mathrm{gi}}$ são os valores de offset e ganho, è é o ângulo de incidência levando em consideração o elipsoide da Terra. $k$ é a constante temporal aplicada (-68.2). A equação 1 foi aplicada nas imagens do Radarsat (Shepherd, 1997) e a equação 2 nas imagens JERS (Shimada, 1996).

\section{Correção geométrica}

Utilizou-se o método de orto-retificação para correção geométrica e o algoritmo de convolução cúbica na reamostragem. A precisão média final dos pontos teste é de $21 \mathrm{~m}$. Após as etapas de correção, dois processamentos distintos foram realizadas nas imagens (i) determinação dos valores de ó ${ }^{\circ}$ em unidade logarítmica $(\mathrm{dB})$ a partir dos valores em unidade linear e (ii) processamento para segmentação e classificação.

\section{Determinação dos valores de $\sigma^{\circ}$}

Valores médios de $\sigma^{\circ}$ foram determinados para as estacões de coleta de campo. O número médio de pixels 
em cada amostra é de 208 ( \pm 89 SD), o que significa que as médias amostrais apresentam um nível de significância de 95\% de acordo com a estatística para radar e considerando o efeito do "speckel" (Laur et al. 1996).

A partir dos valores de $\sigma^{\circ}, \mathrm{o}$ "índice de biomassa" para cada amostra foi relacionado estatisticamente com os valores de biomassa aérea. Subsequentemente, o modelo estatístico foi avaliado de acordo com e erro quadrático médio (EQM) (Neter et al. 1990).

\section{Segmentação e Classificação - determinação espacial das áreas de plantas aquáticas}

Antes da classificação as imagens foram (i) filtradas com o filtro Gamma (três interações recursivas com uma janela 5x5) para minimizar o efeito do "speckel" (Costa et al. 2002).

$$
\sigma^{o} \text { indice }=\frac{\sigma^{o}{ }_{c+} \sigma^{o}{ }_{L}}{2}
$$

Subsequentemente, as imagens foram (ii) convertidas de 32 bit para 8 bit de acordo com a média e três vezes o desvio padrão da média (99\% da distribuição de níveis digitais originais) para reduzir o tamanho dos arquivos de imagens. A seguir, (iii) máscaras previamente determinadas correspondentes à superfície de água e "terra firme" (região acima da planície de inundação) foram subtraídas das imagens. Estas máscaras foram determinadas através da classificação das imagens adquiridas no período de água baixa e alta, respectivamente. Subsequentemente, (iv) pares de imagens sazonais (JERS e Radarsat) foram submetidos a um processo de segmentação automática de acordo o algoritmo de crescimento de regiões (Bins et al. 1996). O processo de segmentação requer a definição do limiar de área (mínimo número de pixels em uma região) e do limiar de similaridade de nível digital (diferença média do valor de nível digital entre duas regiões) para separação de duas regiões. O limiar de área foi determinado em 50 pixels devido à (i) necessidade de garantir que os dados estatísticos de uma região (definida pelo número de pixels) estão de acordo com os critérios de uma população com distribuição normal para imagens de radar (Laur et al. 1996) e (ii) evitar a segmentação exagerada das imagens. O limiar de similaridade de nível digital foi estabelecido de acordo com o método da diferença significativa de duas médias (Snedecor \& Cochran, 1980), e resultou em um valor de 30. Finalmente, (v) os pares de imagens segmentadas foram submetidos a uma classificação supervisionada de regiões baseada no método da distância de Bahattacharrya (Richards, 1986). A tabela 2 apresenta os números de regiões de treinamento e teste utilizadas para a classificação supervisionada e a matriz de erro da classificação, respectivamente. Detalhes do processo de seleção de

Tabela 1 - Características dos dados de satélite

\begin{tabular}{|c|c|c|c|c|c|c|c|}
\hline Satélite & Aquisiçãa & $\begin{array}{l}\text { Ângulo de } \\
\text { incidência }\end{array}$ & $\begin{array}{c}\text { Cobertura } \\
(\mathrm{km})\end{array}$ & $\begin{array}{l}\text { Modo de } \\
\text { aquisição }\end{array}$ & $\begin{array}{c}\text { Banda/ } \\
\text { polarização }\end{array}$ & $\begin{array}{c}\text { Tamanho } \\
\text { do pixel (m) }\end{array}$ & $\begin{array}{l}\text { Número } \\
\text { de looks }\end{array}$ \\
\hline RADARSAT & $\begin{array}{l}27.05 .96 \\
07.08 .96 \\
11.11 .96 \\
05.04 .97 \\
08.06 .99\end{array}$ & $\sim 43$ & $100 \times 100$ & S6descendente & $\mathrm{C} / \mathrm{HH}$ & $12,5 \times 12,5$ & $1 \times 4$ \\
\hline JERS-1 & $\begin{array}{l}16.05 .96 \\
12.08 .96 \\
22.12 .96 \\
20.03 .97\end{array}$ & $\sim 35$ & $75 \times 75$ & descendente & $\mathrm{L} / \mathrm{HH}$ & $12,5 \times 12,5$ & 3 \\
\hline
\end{tabular}

Tabela 2 - Número de regiões de treinamento e teste para a classificação dentro da planície de inundação

\begin{tabular}{|c|c|c|c|c|c|c|}
\hline \multicolumn{7}{|c|}{ Número de amostras } \\
\hline \multirow{2}{*}{ Imagens classificadas } & \multicolumn{2}{|c|}{ Plantas aquáticas } & \multicolumn{2}{|c|}{ Floresta alagada } & \multicolumn{2}{|c|}{ Floresta não alagada } \\
\hline & Treinamento & Teste & Treinamento & Teste & Treinamento & Teste \\
\hline $\begin{array}{l}\text { RADARSATS6 + JERS, } \\
\text { Novembro }\end{array}$ & 48 & 41 & 10 & 8 & 60 & 29 \\
\hline RADARSATS6 + JERS, Abril & 57 & 49 & 100 & 67 & 20 & 8 \\
\hline RADARSATS6 + JERS, Maio & 122 & 72 & 109 & 71 & - & - \\
\hline RADARSATS6 + JERS, Junho & 76 & 54 & 120 & 64 & - & - \\
\hline RADARSATS6 + JERS, Agosto & 83 & 59 & 97 & 72 & 12 & 8 \\
\hline
\end{tabular}




\section{ACTA AMAZONICA}

regióes podem ser encontrados em Costa (2004).

\section{RESULTADOS}

\section{Biomassa de plantas aquáticas}

A tabela 3 apresenta os valores medidos de biomassa seca aérea, submersa e total para H. amplexicaulis, a espécie mais comum na área de estudo (65\% das amostras). A biomassa submersa corresponde à biomassa do talo submerso e a biomassa da raiz $(5 \%$ da biomassa total de acordo com Junk \& Piedade (1997).

De uma forma geral, a biomassa das plantas aumentou de forma constante de novembro até agosto, seguindo a subida e descida dos níveis de água. De fato, as diferenças sazonais de biomassa são principalmente devido ao aumento da biomassa submersa. A biomassa submersa contribui para a biomassa total o correspondente a $52 \%, 88 \%, 86 \%, 81 \%$ e $83 \%$, respectivamente, para novembro, abril, maio, junho e agosto. Esta alta porcentagem é uma estratégia de sobrevivência desta espécie para acompanhar a subida da água. Estratégia similaré observada para outras espécies de gramíneas fixas ao substrato da Amazônia (Piedade et al. 1991).

\section{Imagens de radar}

\section{Determinação dos valores de retroespalhamento para estimativas de biomassa}

O modelo estatístico gerado, $\log B a=4,002+0,175 \times \sigma^{o}$ indice, resultou em um coeficiente de correlação igual a 0,82 . Os resultados do modelo estatístico são apresentados na tabela 4 .

Tabela 3 - Valores médios de biomassa seca aérea, submersa e total de $H$. amplexicaulis

\begin{tabular}{lccc}
\hline \hline \multirow{2}{*}{ Mes } & \multicolumn{3}{c}{ H. amplexicaulis } \\
\cline { 2 - 4 } & Aérea & Submersa & Total \\
\hline Novembro & $91(93)$ & $100(103)$ & $191(197)$ \\
Abril & $270(72)$ & $1985(257)$ & $2255(314)$ \\
Maio & $347(256)$ & $2226(517)$ & $2573(448)$ \\
Junho & $556(226)$ & $2378(653)$ & $2934(674)$ \\
Agosto & $590(200)$ & $2885(52)$ & $3489(210)$ \\
\hline \hline
\end{tabular}

Os valores estão em gm $^{-2}$ de biomassa seca; valores entre parênteses correspondem a um desvio padrão da média. Valores de biomassa submersa estão ajustados de acordo com a diminuição do nível da água.
O teste do modelo estatístico foi realizado com $25 \%$ das amostras do modelo e avaliado de acordo com o erro quadrático médio (EQM) (Neter et al. 1990). Os valores de EQM (modelo) e EQM* (teste do modelo) são similares, o que demostra que o modelo estatístico é estável e pode ser utilizado para as estimativas de biomassa.

A curva do modelo estatístico é apresentada na figura 3. A curva sugere a existência de um ponto de saturação para os valores de ó ${ }^{\circ}$. Baseado na solução da segunda derivada da curva do polinômio de terceira ordem, definiu-se o ponto de saturação da biomassa aérea em $620 \mathrm{gm}^{-2}$. A definição deste ponto é importante para entender a capacidade de estimativa do modelo estatístico, porque acima deste valor as estimativas de biomassa aérea através do modelo não são confiáveis.

Pontos de saturação similares $\left(400 \mathrm{gm}^{-2}\right)$ foram determinados para estimativa de biomassa aérea de arroz em campos alagados utilizando imagens de radar do ERS-1 (Le Toan et al. 1997). Contudo, estimativas utilizando imagens do Radarsat adquirida com ângulo de incidência menor $\left(23^{\circ}\right)$ resultou em um ponto de saturação inferior $\left(200 \mathrm{gm}^{-2}\right)$, para o mesmo tipo de cultura de arroz (Ribbes \& Le Toan, 1999). Estas diferenças podem estar relacionadas com a estrutura de plantio de arroz quando comparado com o processo natural de crescimento de plantas aquáticas na Amazônia. O plantio de arroz estudado por Ribbes \& Le Toan (1999) apresenta uma distribuição de 20 agregados de plantas por $\mathrm{m}^{-2}$, distribuídos em fileiras. Na área de estudo, H. amplexicaulis não forma agregados, a densidade é em média 111 talos $\mathrm{m}^{-2}$ (Costa, et al. 2002), e não existe uma orientação horizontal preferencial de crescimento. Também, a complementaridade das bandas C e L

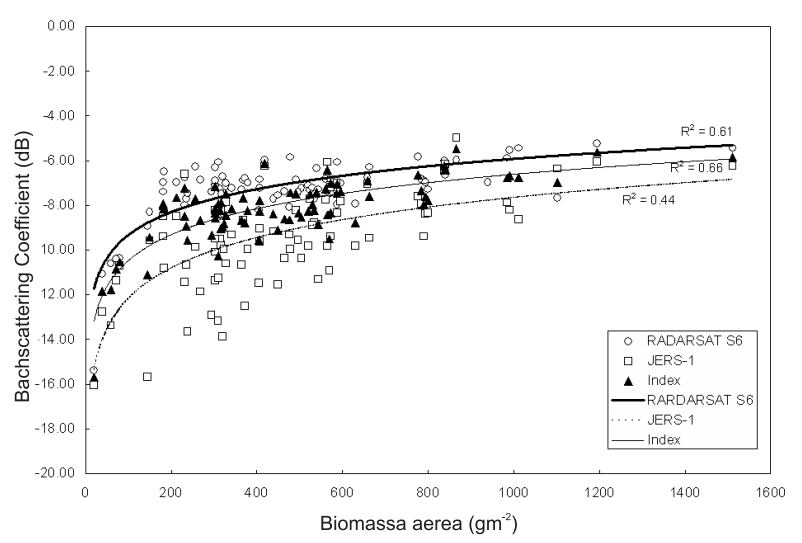

Figure 3 - Variação do retroespalhamento $(\mathrm{dB})$ de acordo com a biomassa seca aérea para $H$. amplexicaulis.

5 km - (a) Novembro, 1996 -água baixa · 5 km - (b) Abril, 1997 - subida da água

Tabela 4 - Resultados estatísticos do modelo de cálculo de biomassa aérea

\begin{tabular}{|c|c|c|c|c|c|c|c|c|c|}
\hline Variável & $\begin{array}{l}\text { Número de } \\
\text { amostras }\end{array}$ & Média & $\begin{array}{l}\text { Desvio } \\
\text { padrão }\end{array}$ & $\begin{array}{c}\text { Coeficiente } \\
\text { de correlação }\end{array}$ & $\begin{array}{c}\text { Coeficiente } \\
\text { de determinação }\end{array}$ & EQM & Error & $\begin{array}{c}\text { Nível de } \\
\text { Significancia }\end{array}$ & $E Q M^{*}$ \\
\hline $\begin{array}{l}\text { Log da } \\
\text { biomassa seca }\end{array}$ & 80 & 2,6 & 0,33 & 0,82 & 0,67 & 206 & $34 \%$ & $p<0,05$ & 236 \\
\hline
\end{tabular}

EQM*é o erro quadrático médio para o teste do modelo. 
(este estudo) deve influenciar na determinação de um ponto de saturação mais alto para biomassa aérea.

\section{Segmentação e Classificação - deter- minação espacial das áreas coberta por plantas aquáticas}

De uma forma geral, o método de classificação resultou em uma precisão média maior que $95 \%$ para os pares de imagens (Radarsat e JERS) sazonais (Tabela 5). As áreas de planta aquática foram corretamente classificadas em mais de $95 \%$ dos casos para qualquer estação do ano. A maior confusão na classificação (11\%) foi observada entre as classes de floresta inundada e floresta não inundada em novembro (inundada sazonalmente). Possivelmente o alto conteúdo de umidade do solo, devido ao começo da subida de água, resultou em valores de retroespalhamento altos, os quais favorecem a confusão com a classe de floresta inundada. Os mapas temáticos sazonais são apresentados na figura 4 .

\section{Cálculo da produção primária líquida}

A PPL foi calculada para cada célula de resolução da imagem (pixel) considerando (i) a área classificada de plantas aquáticas (item 3.2.3), (ii) o algoritmo estatístico para determinação de biomassa seca aérea (item 3.2.1), (iii) a porcentagem de biomassa seca submersa na biomassa seca total (item 3.1) e (iv) a produção de biomassa líquida mensal. A estratégia para determinação de cada um destes parâmetros é apresentada a seguir.

\section{(i)Separação de áreas sazonais corres- pondentes a plantas aquáticas}

A partirdos mapas temáticos resultantes da classificação, máscaras sazonais correspondentes a plantas aquáticas foram determinadase sobrepostas nos pares sazonais de imagens de radar.

\section{(ii)Geração de imagens de biomassa seca aérea $\left(B_{a}\right)$}

Imagens de biomassa seca aérea foram calculadas aplicandose o modelo estatístico nas imagens sazonais do Radarsat e JERS correspondentes aárea coberta por plantas aquáticas (item anterior).

\section{(iii)Geração de imagens de biomassa seca total $\left(B_{t}\right)$}

A biomassa seca total foi calculada para cada pixel da imagem de biomassa seca aérea (item anterior) considerando a porcentagem de biomassa seca submersa na biomassa seca total. Imagens de biomassa total foram geradas para novembro, abril, maio, junho e agosto. $\mathrm{O}$ cálculo da biomassa total para cada mês $\left(\mathrm{B}_{\mathrm{T}}\right)$ é o somatório da biomassa de todos os pixels da imagem de biomassa

Tabela 5 - Matriz de confusão da classificação

\begin{tabular}{|c|c|c|c|}
\hline \multicolumn{4}{|c|}{ Classe verdadeira } \\
\hline \% Classificada como & Planta aquática & Floresta alagada & Floresta não alagada \\
\hline \multicolumn{4}{|c|}{ RADARSAT S6 + JERS-1, Novembro - precisão geral = 97,02\% } \\
\hline Planta aquática & 99,1 & 0 & 0,91 \\
\hline Floresta alagada & 0 & 58,9 & 41, \\
\hline Floresta não alagada & 3,3 & 11,3 & 85 \\
\hline \multicolumn{4}{|c|}{ RADARSAT S6 + JERS-1, Abril - precisão geral = 96,94\% } \\
\hline Planta aquática & 94,4 & 5,6 & 0 \\
\hline Floresta alagada & 0,9 & 97,8 & 1,2 \\
\hline Floresta não alagada & 0 & 2,9 & 97,1 \\
\hline \multicolumn{4}{|c|}{ RADARSAT S6 + JERS-1, Maio - precisão geral = 97,39\% } \\
\hline Planta aquática & 95,3 & 4,5 & - \\
\hline Floresta alagada & 0 & 100 & - \\
\hline Floresta não alagada & - & - & - \\
\hline \multicolumn{4}{|c|}{ RADARSAT S6 + JERS-1, Junho - precisão geral = 95,47\% } \\
\hline Planta aquática & 97,4 & 2,6 & - \\
\hline Floresta alagada & 0,6 & 99,36 & - \\
\hline Floresta não alagada & - & - & - \\
\hline \multicolumn{4}{|c|}{ RADARSAT S6 + JERS-1, Agosto - precisão geral = 93,85\% } \\
\hline Planta aquática & 93,4 & 5,5 & 1,11 \\
\hline Floresta alagada & 4,6 & 95,5 & 0 \\
\hline Floresta não alagada & 0 & 8,1 & 91,9 \\
\hline
\end{tabular}




\section{ACTA AMAZONICA}
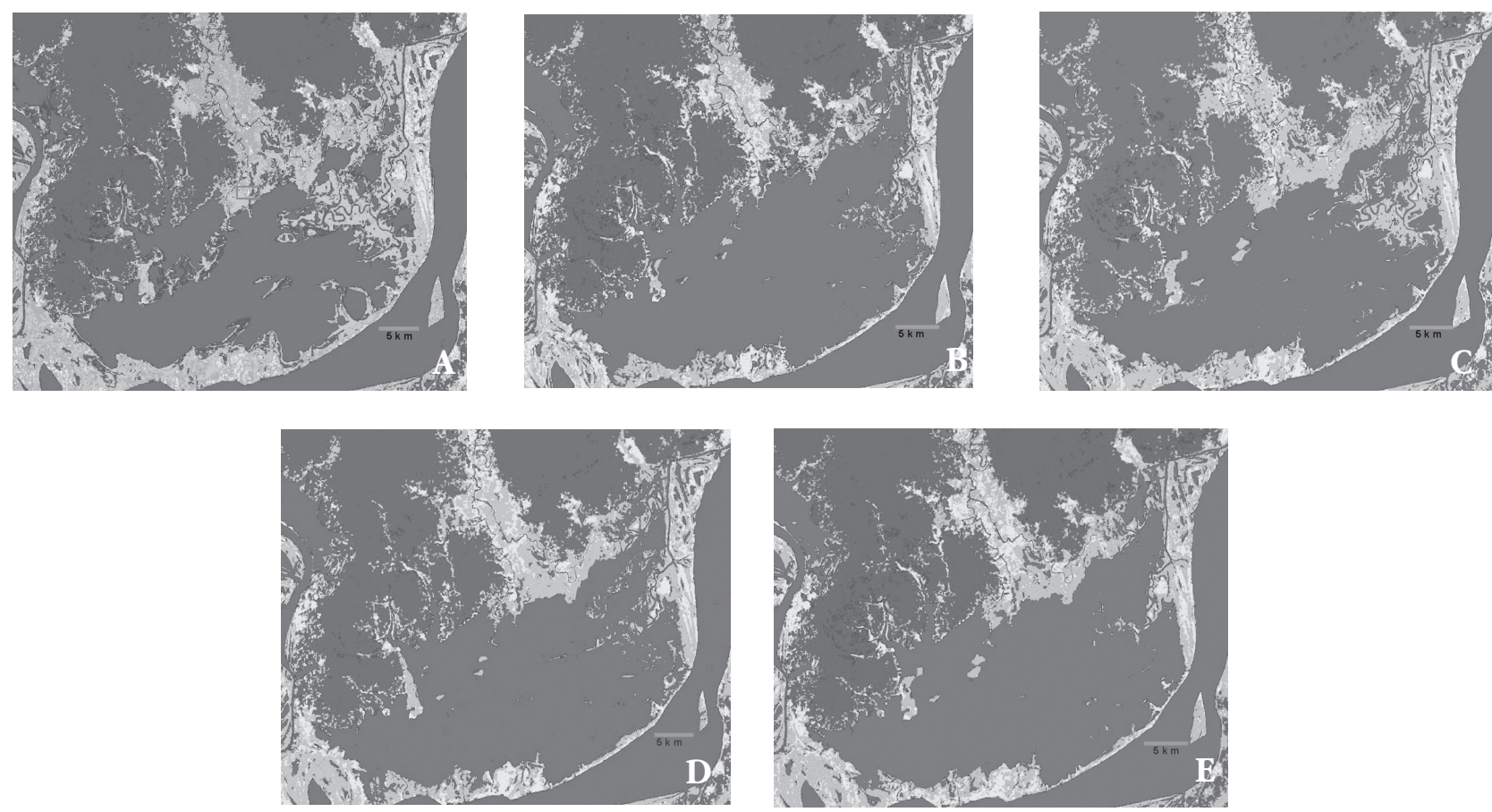

Figura 4 - Mapas temáticos gerados a partir da classificação. AZUL CLARO= plantas aquáticas; AMARELO=floresta alagada; LARANJA=floresta não alagada sazonalmente; VERDE=floresta de "terra firme" (floresta, pasto e cerrado); AZUL= água.

total do respectivo mês. A tabela 6 apresenta os valores de $\mathrm{B}_{\mathrm{T}}$ calculados para novembro, abril, maio, junho e agosto. Os valores de $\mathrm{B}_{\mathrm{T}}$ calculados são estatisticamente semelhantes $(\mathrm{p}<0,05)$ aos $\mathrm{B}_{\mathrm{T}}$ calculados por meio de medidas de campo; contudo, observa-se uma diferença de $28 \%$ entre estes valores. Note que esta diferença é influenciada principalmente pela maior discrepância entre as $\mathrm{B}_{\mathrm{T}}$ de novembro (54\% de diferença), quando os valores de $\mathrm{B}_{\mathrm{T}}$ são os mais baixos e, portanto, menos significantes para a estimativa de produção primária líquida total. Desconsiderando-se a discrepância observada para novembro, a diferença total entre a $B_{T}$ medida em campo e a $B_{T}$ estimada com imagens de satélite diminui para $21 \%$.

Os resultados mostram que a $B_{T}$ aumenta continuamente a partir de novembro, quando a nova geração de plantas aquáticas começa a se desenvolver resultando em uma biomassa seca total de $0,1 \times 10^{12} \mathrm{~g}$. As folhas e talos se formam e crescem rapidamente mantendo as estruturas fotossintéticas acima do nível da água. Em maio, no máximo nível da água, a biomassa seca total é $1,45 \times 10^{12} \mathrm{~g}$ em uma área de $395 \mathrm{~km}^{2}$. A partir de maio o nível da água começa a descer arrastando algumas plantas para o Rio Amazonas. Consequentemente em agosto, a área ocupada pelas plantas e a biomassa seca total decrescem para $281 \mathrm{~km}^{2} \mathrm{e} 0,95 \times 10^{12} \mathrm{~g}$, respectivamente. Valores similares de biomassa seca total mensal são observados para outras espécies em outras regiões da Amazônia (Piedade et al. 1992).

(iv)Estimativa de biomassa mensal
Como reportado anteriormente, nossos dados foram adquiridos em cinco meses distintos do ciclo de crescimento das plantas, o qual começa em novembro/dezembro, com a subida das águas, maio/junho, com o pico de máximo nível de água, até setembro, quando as plantas estão na fase de senescência. Contudo, como já havia salientado Piedade et al. (1991), estimativas mensais são importantes para estudos de PPL de plantas aquáticas na Amazônia devido ao crescimento contínuo das plantas em um ciclo hidrológico. Portanto, a biomassa total $\left(\mathrm{B}_{\mathrm{T}}\right)$ dos meses nos quais não se obteve dados (campo e satélite) foi estimada estatisticamente a partir da biomassa total calculada com dados de campo e satélite. Utilizou-se a curva de mínimos quadrados determinada com os $\mathrm{B}_{\mathrm{T}}$ medidos $\left(\mathrm{R}^{2}=0,99 ; \mathrm{n}=5\right.$; $\mathrm{p}<0,01)$ para determinação da biomassa total estimada correspondente aos meses de dezembro, janeiro, fevereiro, março e julho. Este procedimento foi considerado satisfatório porque um contínuo aumento da biomassa de plantas aquáticas é observado para várias regiões da Amazônia (Piedade et al. 1992; Junk \& Piedade, 1997). A mesma relação estatística foi utilizada nas imagens de satélite (imagens $\mathrm{B}_{\mathrm{t}}$ item anterior) para estimar imagens de $B_{t}$ para dezembro, janeiro, fevereiro, março e julho. A tabela 6 apresenta os valores de biomassa total $\left(\mathrm{B}_{\mathrm{T}}\right)$ estimados.

\section{(v)Estimativa de PPL}

A produção primária líquida é o incremento da biomassa total e biomassa perdida entre dois meses consecutivos para cada pixel 


\section{ACTA AMAZONICA}

USO DE IMAGENS DE RADAR PARA O CÁLCULO DA PRODUCÃO PRIMÁRIA DE PLANTAS AQUÁTICAS NAS VÁRZEAS DA AMAZÔNIA. da imagem. Caso não haja incremento da biomassa total (diminuição ou constância) entre dois meses consecutivos, a PPL é determinada apenas em função da biomassa perdida entre dois meses consecutivos (Junk \& Piedade, 1993). Matematicamente, a PPLé calculada por meio das seguintes equações:

$$
\begin{aligned}
& P P L_{m i=}\left(B_{(t+1) i}-B_{t i}\right)+\left(L_{(t+1)} \times B_{(t+1) i}\right) \quad \Delta \mathrm{B}_{\mathrm{ti}}>0 \quad \text { Equação4 } \\
& P P L_{m i=}\left(L_{(t+1)} \times B_{(t+1) i}\right) \quad \Delta \mathrm{B}_{\mathrm{ti}} \leqslant 0 \quad \text { Equação5 }
\end{aligned}
$$

Onde PPL $\mathrm{mi}_{\mathrm{i}}$ é a produtividade mensal, $\mathrm{B}_{\mathrm{ti}}$ e $\mathrm{B}_{(\mathrm{t}+1) \mathrm{i}}$ são as biomassas totais de dois meses consecutivos, $\mathrm{t} \mathrm{e}(\mathrm{t}+1), \mathrm{L}_{(\mathrm{t}+1)}$ é a biomassa perdida durante dois meses consecutivos e corresponde a $25 \%$ de $B_{(t+1) \mathrm{i}}$ (Junk \& Piedade, 1993) e " $\mathrm{B}_{\mathrm{ti}}$ é o incremento na biomassa total entre dois meses consecutivos.

Calculada a PPL ${ }_{\text {mi }}$ a produtividade primária líquida total (PPL $)$, para cada pixels da imagem, é a soma das PPL $_{\text {mi }}$ de cada pixel. A tabela 7 apresenta os valores de PPL média e total por ano estimada a partir de dados de satélite. A partir da imagem de $\mathrm{PPL}_{\mathrm{i}}$ e da porcentagem de carbono em $H$. amplexicaules ( $46 \% \mathrm{da}$ biomassa total, (Costa, 2000)), calculou-se a imagem de carbono total assimilado em um ano do ciclo hidrológico (Figure 5).

\section{DISCUSSÃO}

A tabela 8 mostra valores de máxima biomassa para plantas aquáticas enraizadas da Amazônia reportados por vários autores. De uma forma geral os valores variam de aproximadamente $5000 \mathrm{a} 8000 \mathrm{gm}^{-2}$. Os valores mais altos são para E. polystachya $\left(8000 \mathrm{gm}^{-2}\right)$, uma espécie que suporta condições de alto stress ambiental (Piedade et al. 1991). Valores relativamente mais baixos são reportados para Paspalum fasciculatum e H. amplexicaulis, espécies com um menor porte quando comparadas com E. polystachya. O método baseado em dados de radar estimou uma biomassa máxima de 4500gm

Tabela 6 - Comparação da biomassa total estimada por meio de dados de campo e imagens de satélite

\begin{tabular}{lcc}
\hline \hline & $\begin{array}{c}\text { Biomassa total }(\mathrm{g}) \\
\text { Campo e satélite * }\end{array}$ & $\begin{array}{c}\text { Biomassa Total } \\
(\mathrm{g}) \text { satélite }\end{array}$ \\
\hline Novembro & $0,65 \times 1011$ & $1 \times 1011$ \\
Dezembro & $1,4 \times 1011^{* *}$ & $1,2 \times 1011$ \\
Janeiro & $2,3 \times 1011^{* *}$ & $2,1 \times 1011$ \\
Fevereiro & $3,2 \times 1011^{* *}$ & $2,9 \times 1011$ \\
Março & $4 \times 1011^{* *}$ & $3,6 \times 1011$ \\
Abril & $5 \times 1011$ & $6,1 \times 1011$ \\
Maio & $10.2 \times 1011$ & $14,5 \times 1011$ \\
Junho & $8,7 \times 1011$ & $7,1 \times 1011$ \\
Julho & $9,5 \times 1011^{* *}$ & $7,1 \times 1011$ \\
Agosto & $9,8 \times 1011$ & $9,5 \times 1011$ \\
\hline \hline
\end{tabular}

* são valores estimados baseados na biomassa medida em campo e a área de plantas aquáticas determinada com imagens de satélites; ${ }^{* *}$ são valores estimados.
${ }^{2}$ para $H$. amplexicaulis, a qual esta dentro dos valores medidos para outras espécies da Amazônia utilizando intensa amostragem de campo. Esta mesma comparação é valida para a estimativa de PPL pelo método de radar e estimativas baseadas em coletas de campo, como reportado no item anterior.

Os valores de PPL média estimada $\left(6350 \mathrm{gm}^{-2} \mathrm{yr}^{-1}\right)$ ou uma PPL anual de $4,1 \times 10^{12}$ gyr $^{-1}$, para uma área máxima de plantas aquáticas de $394 \mathrm{~km}^{2}$ (Tabela 7) são semelhantes às estimativas apresentadas para outras espécies de plantas aquáticas na Amazônia. Por exemplo, $9930 \mathrm{gm}^{-2} \mathrm{yr}^{-1}$ para E. polystachya (Piedade et al. 1991) e $2700 \mathrm{gm}^{-2} \mathrm{em}$ quatro meses para Oryza perennis (Junk \& Piedade, 1993). As diferenças em PPL para cada espécie estão possivelmente relacionada com as estratégias necessárias para sobreviverem em um determinado habitat e as características da espécie. Por exemplo, $E$. polystachya coloniza regiões de alto stress ambiental devido à correnteza e grandes variações de nível de água $(8 \mathrm{~m})$. Para sobreviver nestas condições esta espécie apresenta rápido crescimento como resultado da uma alta eficiência em converter a radiação solar interceptada em biomassa. Esta e uma característica típica de plantas C4 (Piedade et al., 1991). Por outro lado, áreas mais protegidas e com menor variação de nível de agua (4m), como no caso da região de estudo, são colonizadas por $H$. amplexicaulis, uma espécie que apesar do menor porte e do aparente modo menos eficiente de fotossintetizar por ser uma planta C3 (Costa, 2000), apresenta valores de PPL altos. Esta alta PPL ocorre parcialmente devido à alta densidade destas plantas (111 plantas $\mathrm{m}^{-2}$, Costa et al., 2002) comparada com a densidade de E. polystachya (30 plantas $\mathrm{m}^{-2}$, Costa et al. 2002) e uma possível adaptação para condições de máxima luz solar resultando em um aumento nas taxas fotossintéticas, o que caracteriza as plantas $\mathrm{C} 4$. Juncus roemerianus, uma espécie C3 característica da América do Norte, apresenta este mesmo tipo de adaptação (Mitsch \& Gosselink, 2000). Portanto, na planície de inundação da

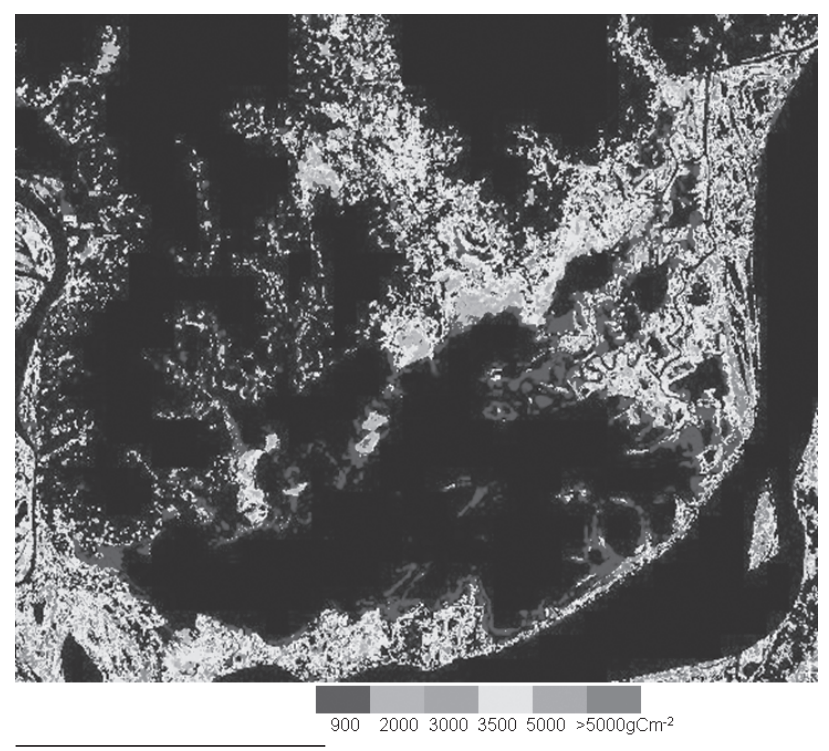

Figura 5 - Distribuição espacial da produção anual de carbono por plantas aquáticas. 


\section{ACTA AMAZONICA}

Amazônia, tanto as plantas aquáticas C3 (este estudo) como as C4 (Piedade et al. 1991), de formas distintas, apresentam estratégias para alcançar alta produtividade.

\section{CONCLUSÕES}

Imagens dos satélites Radarsat e JERS foram utilizadas eficientemente para o estimar a produtividade primária de plantas aquáticas na planície de inundação da Amazônia. A produção primária é estimada a partir da determinação espacial da área ocupada por plantas aquáticas e da relação estatística entre o "índice de biomassa" () e valores de biomassa amostrados em campo. As áreas de plantas aquáticas foram mapeadas com precisão maior que $95 \%$ pelo método de classificação supervisionada por regiões. Este método (segmentação/classificação) foi pela primeira vez utilizado para mapeamento da planície de inundação da Amazônia. A relação estatística para determinação de biomassa aérea apresentou um coeficiente de correlação de $0,82(p<0,05)$ e um ponto de saturação de biomassa aérea em $620 \mathrm{gm}^{-2}$. Acima do valor de saturação de biomassa as estimativas do modelo estatístico não são confiáveis. Contudo, a máxima biomassa média aérea observada (agosto, 1996) está abaixo do valor de saturação calculado, o que torna o modelo estatístico confiável para estimativas de biomassa aérea para a região de estudo.

De uma forma geral, a estimativa de PPL pelo método de radar apresenta uma boa concordância com os valores medidos em campo e valores reportados por outros autores, e permitiu estimar uma produção de carbono anual de $2920 \mathrm{~g} \mathrm{C} \mathrm{m}^{-2} \mathrm{yr}^{-1} \mathrm{ou}$ um total de $1,9 \times 10^{12} \mathrm{~g} \mathrm{C} \mathrm{yr}^{-1}$ para a área de estudo coberta por plantas aquáticas (máximo em maio - $394 \mathrm{~km}^{2}$ ). Espacialmente (Figura 5), os valores mais baixos de carbono $\left(<900 \mathrm{gCm}^{-2}\right)$ são observados em regiões onde as plantas se desenvolveram somente no começo do ciclo de crescimento. Valores mais altos de carbono $\left(>5000 \mathrm{gCm}^{-2}\right)$ são observados em regiões próximas ao Rio Amazonas onde a influência de água rica em nutrientes é maior e, portanto uma alta produtividade é observada.

O método baseado em imagens de radar é uma alternativa confiável para estudos de produtividade de plantas aquáticas emergentes em escala local e regional, porque fornece informação com a resolução espacial (pixel) das imagens de satélite comparado com as extrapolações espaciais necessárias quando se utiliza método de amostragem de campo tradicional ou modelo baseado em processos fisiológicos.

\section{AGRADECIMENTOS}

A Canadian International Space Agency e Fundação de Amparo a Pesquisa do Estado de São Paulo pelos recursos financeiros para conduzir os trabalhos de campo. Contribuições financeiras do projeto LBA coordenado pelo Dr. John Melack são também reconhecidas. A Coordenação de Aperfeiçoamento de Pessoal de Nível Superior (CAPES) forneceu a bolsa de estudo para a autora. A Japonesa Space Agency e a Canadian Space Agency forneceram as imagens dos satélites JERS-1 e Radarsat, respectivamente. Gostaria de agradecer aos vários colegas e amigos que ajudaram durante o trabalho de campo, Dra. Novo, Dr. Mantovani e Gilson. Agradeço também a revisão detalhada deste manuscrito por parte de um dos revisores.

\section{BIBLIOGRAFIA CITADA}

Bins, L.S.; Fonseca, L.M.G.; Eethal, G.J. ;Mitsuo, F. 1996. Satellite imagery segmentation: a region growing approach. Proceedings. CD. In: Simpósio Brasileiro de Sensoriamento Remoto. Salvador, Bahia, Brasil.

Costa, M.P.F. 2000. Net Primary Productivity of Aquatic Vegetation of the Amazon Floodplain: a Multi-SAR Satellite Approach. Ph.D. Thesis, University of Victoria, Victoria, Canada. 233p.

Costa, M.P.F. 2004. Use of SAR satellite for mapping zonation of vegetation communities in the Amazon floodplain. International Journal of Remote Sensing. 25(10):1817-1835.

Costa, M.P.F., Niemann, O., Novo, E., Ahern, F. 2002. Biophysical Properties and mapping of aquatic vegetation during the hydrological cycle of the Amazon floodplain using JERS-1 and RADARSAT, 2001. International Journal of Remote Sensing. 23(7):1401-1260.

Downing, J.A.; Anderson, M.R. 1985. Estimating the standing

Tabela 7 - PPL média e total estimada a partir de dados de satélite e a partir de dados de campo

\begin{tabular}{lccc}
\hline \hline & NPP & \\
\hline Média $(\mathrm{gm}-2)$ satélite & Total $(\mathrm{g}$ yr-1)satélite & Média $(\mathrm{gm}-2)$ campo & Total ( gyr-1)Campo + satélite* \\
$6350( \pm 1778)$ & $4.1 \times 1012( \pm 1,1 \times 1012)$ & 8430 & $5,4 \times 1012$ \\
\hline \hline
\end{tabular}

*valores calculados a partir da PPL média (dados de campo) e área de plantas aquáticas determinada com imagens de satélite. Os valores entre parêntese correspondem a um erro de $28 \%$ associado à diferença média entre valores determinados com dados de campo e com as imagens de satélite.

Tabela 8 - Valores de máxima biomassa seca total para diferentes espécies da planície de inundação

\begin{tabular}{lccc}
\hline \multicolumn{1}{c}{ Espécie } & Localização & Biomassa máxima & Autor \\
\hline E. polystachya & Amazonia central & 6880 & Piedade et al., 1991 \\
P. fasciculatum & Amazonia central & 5760 & Junk and Piedade, 1993 \\
H. amplexicaulis & Amazonia central & 2270 & Junk and Piedade, 1997 \\
H. amplexicaulis & Baixo Amazonas & $4553^{*}$ & Este estudo \\
E. polystachya & Baixo Amazonas & 4670 & Costa, 2000 \\
\hline
\end{tabular}

* valor calculado por meio de imagens de satélite; $3490 \mathrm{gm}^{-2}$ é a biomassa máxima total calculada com dados de campo. 
biomass of aquatic macrophytes. Canadian Journal of Fisheries and Aquatic Science, 42:1860-1869.

Junk, W.J. 1993. Wetlands of Tropical South America. In: Whigham D.F., Hejny, S., Dykyjova, D. (Eds). Wetlands of the world. IC. Kluwer, Dordrecht, pp: 679-739.

Junk, W.J.; Piedade, M.T. 1993. Biomass and primary productivity of herbaceous plant communities in the Amazon floodplain. Hydrobiology, 263: 155-162.

Junk, W.J.; Piedade, M.T. 1997. Plant life in the floodplain with special reference to herbaceous plants. In: W.J. Junk (Eds.). The Central Amazon Floodplain: Ecology of a Pulsing System. Springer. Berlin. pp.147-181.

Laur, H., Bally, P., Meadows, P., Sanchez, J., Schaettler, B.,; Lopinto, E. 1996. Derivation of the backscattering coefficient $s^{\circ}$ in ERS.SAR.PRI products. ESA/ESRIN, Issue 2, Rev. 2. 55p.

Le Toan, T., Ribbes, F., Wang, L., Floury, N., Ding, K.; Kong, J.A.,; Fujita, M. 1997. Rice crop mapping and monitoring using ERS-1 data based on experiment and modeling results. IEEE Transaction of Geoscience and Remote Sensing, 35(1): 41-55.

Melack, J.M.; Forsberg, B.R. 2000. Biogeochemistry of Amazon floodplain lakes and associated wetlands. In: M.E. McClain, R.L. Victoria, J.E. Richey (Eds.) Biogeochemistry of the Amazon Basin. Oxford University Press. Oxford. pp.210-260.

Mitsch, J.W.; Gosselink, J.G. 2000. Wetlands. John Wiley \& Sons, Inc, New York, USA. 920p.

Neter, J.; Wasserman, W.; Kutner, M.H. 1990. Applied Linear Statistical Models. IRWIN. USA. 1181p.

Novo, E.M.L.M.; Leite, F.A.; Avila, J.; Ballester, V.; Melack, J.M. 1997. Assessment of Amazon floodplain habitats using TM/ Landsat data. Ciência e Cultura. 49(4):280-284.

Piedade, M.T.F.; Junk, W.J.; Long, S.P. 1991. The productivity of the C4 grass Echinochloa polystachya on the Amazon floodplain. Ecology. 72(4), 1456-1463.

Piedade, M.T.F.; Junk, W.J.; Mello, J.A.N. 1992. A floodplain grassland on the central Amazon. In: B.J.Jones and M.J. Roberts (Eds). Primary Productivity of Grass Ecosystems of the Tropics and Sub-tropics. Chapman \& Hall/UNEP. New York. pp.127-158.

Potter, C.S.; Randerson, J.T.; Field, C.B.; Matsaon, P.A.; Vitousek, P.M.; Mooney, H.A.; Klooster, S.A. 1993. Terrestrial ecosystem production: a process model based on global satellite and surface data. Global Biogeochemical cycles. 7(4):811-841.

RADAMBRASIL. 1976. Folha SA.21-Santarém. Levantamento de Recursos Naturais. Vol.10. Rio de Janeiro, Brasil. 200p.

Ribbes, F.; Le Toan, T. 1999. Rice field mapping and monitoring with RADARSAT data. International Journal of Remote Sensing, 20(4), 745-765.

Richards, J.A. 1986. Remote Sensing Digital Analysis. SpringerVerlag. Berlin. 281p.

Sellers, P.J.; Meeson, B.W.; Hall, F.G.; Asrar, G; Murphy, R.E.; Schiffer, R.A; Bretherton, F.P.; Dickinson, R.E.; Ellingson, R.G.; Field, C.B.; Huemmrich, K.F.; Justice, C.O.; Melack, J.M.; Roulet, N.T.; Schimel, D.S.; \& Try, P.D. 1995. Remote Sensing of the land surface for studies of global change: models-algorithms-experiments. Remote Sensing of Environment. 51(1):3-26.

Shepherd, N. 1997. Output scaling look up tables in RADARSAT CDPF products. In: Proceedings of a Workshop on RADARSAT Data Quality. Quebec, Canada. 20p.

Shimada, M. 1996. Radiometric and geometric calibration of JERS-1 SAR. Advances in Space Research, 17: 79-88.

Snedecor, G.W.; Cochran, W.G. 1980. Statistical Methods. Iowa State University Press. Iowa, USA. 507p.

RECEBIDO EM 04/07/2003
ACEITO EM 23/05/2005 